\title{
ACE2 peptide fragment interacts with several sites on the SARS-CoV-2 spike protein S1
}

\author{
Aleksei Kuznetsov \\ University of Tartu \\ Piret Arukuusk \\ University of Tartu \\ Heleri Heike Härk \\ University of Tartu \\ Erkki Juronen \\ Icosagen Cell Factory OÜ, Tartu \\ Ülo Langel \\ University of Tartu \\ Mart Ustav \\ Icosagen Cell Factory OÜ, Tartu \\ Jaak Jarv ( $\sim$ jaak.jarv@ut.ee ) \\ University of Tartu
}

\section{Research Article}

Keywords: SARS-CoV-2 spike protein S1, angiotensin-converting enzyme 2 (ACE2)

Posted Date: March 17th, 2021

DOl: https://doi.org/10.21203/rs.3.rs-289393/v1

License: (c) (1) This work is licensed under a Creative Commons Attribution 4.0 International License.

Read Full License 


\section{Abstract}

The influence of the peptide QAKTFLDKFNHEAEDLFYQ on the kinetics of the SARS-CoV-2 spike protein S1 binding to angiotensin-converting enzyme 2 (ACE2) was studied to model the interaction of the virus with its host cell. This peptide corresponds to the sequence $24-42$ of the ACE2 a1 domain, which is the binding site for the S1 protein. The on-rate and off-rate of S1-ACE2 complex formation were measured in the presence of various peptide concentrations using Bio-Layer Interferometry (BLI). The formation of the S1-ACE2 complex was inhibited when the S1 protein was preincubated with the peptide, however, no significant inhibitory effect was observed in the absence of preincubation. Dissociation kinetics revealed that the peptide remained bound to the S1-ACE2 complex and stabilized this complex. This suggestion was confirmed by computational mapping of the $\mathrm{S} 1$ protein surface for peptide binding that revealed two additional sites, located at some distance from the receptor binding domain of $\mathrm{S} 1$. These additional binding sites may affect the interaction between the peptide, the S1 protein, and ACE2.

\section{Introduction}

Binding of SARS-CoV-2 particles to the angiotensin-converting enzyme 2 (ACE2) on host cells leads to the fusion of the virus and host cell membranes and initiates the entrance of the viral RNA into the cells [12]. It has been suggested that blocking this binding process will inhibit the virus entry process and thus may have a therapeutic antiviral effect [3]. The most straightforward way of designing inhibitors is to use peptides to mimic the interaction interface between the spike protein and the ACE2 molecule in complex [4-6]. The spike protein is composed of S1 and S2 domains, and it is S1 that contains the receptorbinding domain (RBD) that binds to ACE2 [7-8]. As the molecular structure of the S1-ACE2 complex is known [7-9], and the atomic coordinates and experimental data (code 6LZG) have been deposited in the PDB database (www.wwPDB.org), inhibitory peptides can be designed based on the structure of the complex. However, as ACE2 is a physiologically important enzyme, its inhibition by antiviral prophylaxis with peptides derived from the spike protein is not a promising approach. Therefore, we designed peptides that are derived from the ACE2 structure and interact with the RBD of the spike protein S1 $[5,6]$.

Initially, the ACE2 binding site on the S1 protein was mapped computationally [5]. This analysis revealed that the peptide STIEEQAKTFLDKFNHEAEDLFYQSSL, derived from the a1 domain of the N-terminal part of ACE2, containing amino acids 19-45, can be truncated from both ends without significant loss of binding. Therefore, the shorter peptide QAKTFLDKFNHEAEDLFYQ (amino acids 24-42), which still interacts effectively with the $S 1$ protein (docking energy $E_{\text {dock }}=-11.7 \mathrm{kcal} / \mathrm{mol}[5]$ ), was selected for experiments in this study. The molecular mass of this peptide is too low for a direct binding assay using Bio-Layer Interferometry technology (BLI) [10], and it is unclear how chemical modification or loading with a cargo molecule or linker group would influence the binding properties of the peptide. Therefore, we loaded ACE2 onto the biosensors and studied the influence of the peptide on the kinetics of the S1-ACE2 interaction.

\section{Results And Discussion}




\section{Kinetic measurements of the S1-ACE2 binding interaction}

The influence of the peptide QAKTFLDKFNHEAEDLFYQ on the binding of the SARS-CoV-2 spike S1 protein with ACE2 was investigated by loading ACE2 onto biosensors, then dipping into a buffer containing S1 protein, or S1 protein and peptide. This experimental setup allowed characterization of the complex formation and dissociation reactions, described by the ascending and descending parts of the graphs, respectively, exemplified in Fig 1. Taking the ascending part of the plot, the complex formation reaction is characterized by the first-order rate constant $k_{o n}\left(s^{-1}\right)$ and the second-order rate constant $k_{\text {ass }}$ $\left(\mathrm{M}^{-1} \mathrm{~s}^{-1}\right)$. In the latter case, the concentration of the $\mathrm{S} 1$ protein in the assay buffer is taken into consideration [10]. The descending part of the plot allows calculation of the complex dissociation rate constant, denoted here as $\mathrm{k}_{\text {off }}\left(\mathrm{s}^{-1}\right)$. The equilibrium constant, $\mathrm{K}_{\mathrm{D}}$, for the complex dissociation can be calculated as the ratio of the $k_{\text {off }}$ and $k_{\text {ass }}$ values $[10,11]$. In this study, the $K_{D}$ values for the S1-ACE2 complex, calculated from two parallel experiments in the absence of the peptide, were $(1.28 \pm 0.01) 10^{-8} \mathrm{M}$ and $(3.05 \pm 0.01) 10^{-8} \mathrm{M}$, respectively. These values agree with the $K_{D}=2.9 \times 10^{-8} \mathrm{M}$, published by Reaction Biology [12], and confirm the reliability of the assay procedure.

\section{Peptide influence on the kinetics of the S1-ACE2 interaction}

Figure 1 shows that the time course of interaction of the spike protein S1 with ACE2 (red line) is affected by the addition of $5 \mathrm{mM}$ peptide (green line). For a more detailed analysis of the effect of the peptide on the S1-ACE2 interaction, two series of kinetic experiments were performed, in which varying amounts of the peptide were added to the kinetic assay. One series of experiments simultaneously added the S1 protein and the peptide to the sensor-immobilized ACE2 to initiate the complex formation. In the second series of experiments, preincubation of the $\mathrm{S} 1$ protein with the peptide was performed for 15 minutes before initiation of the complex formation. From these data, the $k_{\text {on }}$ values were calculated, and the results of this analysis are summarized in Figure 2.

Figure 2 reveals that the peptide QAKTFLDKFNHEAEDLFYQ inhibited the rate of S1-ACE2 complex formation, decreasing the rate constant almost two-fold when the spike S1 protein had been preincubated with the peptide. In contrast, no inhibitory effect was observed when the spike S1 protein and peptide were added simultaneously to the assay buffer. These results suggest that the peptide interaction with the spike S1 protein is a slow process, and preincubation is necessary to load the spike S1 protein with the peptide.

Secondly, Figure 2 shows that the rate constant $\left(\mathrm{k}_{\mathrm{on}}\right)$ decreases in the presence of the peptide in a dosedependent manner, and the half-maximal inhibitory effect was reached at $0.7 \pm 0.4 \mathrm{mM}$. Certainly, this value has physical meaning if the peptide-spike S1 protein interaction can be described as an equilibrium process.

Finally, the formation of the S1-ACE2 complex was not completely inhibited by the peptide, since the $\mathrm{k}_{\text {on }}$ values leveled off, even when an excess of the peptide was added. This phenomenon cannot be 
unambiguously explained with the existing data. However, it appears likely that the incomplete inhibition could be connected to the slow rate of the peptide interaction with its binding site on the S1 protein.

\section{Dissociation kinetics of the S1-ACE2 complex}

Dissociation of the ACE2 bound S1 protein was initiated by transferring the biosensor into fresh assay buffer that did not contain peptide or $\mathrm{S} 1$ protein. Therefore, if a binary complex is formed between the ACE2 and S1 proteins, similar $k_{\text {off }}$ values, calculated from the descending part of the kinetic curves (see Figure 1), should describe the dissociation process in experiments, performed at different peptide concentration. However, this was not true of this study, as illustrated in Figure 3.

It is important to emphasize that dissociation of the S1-ACE2 protein complex was initiated by transfer of the biosensor into buffer that did not contain peptide as well as S1. However, it can be seen in Figure 3 that the $k_{\text {off }}$ value depends on the peptide concentration, which was used in the complex formation reaction, demonstrating "memory" relating to the peptide presence in the latter process. These results raise the following questions about the S1-ACE2 complex formation and its structure.

First, the occurrence of such "memory" indicates that different complexes could be formed in the presence of different peptide concentrations, or more likely, the peptide remains in some fraction of this complex, affecting its stability when compared with the binary complex.

Second, half of the peptide's effect on $\mathrm{k}_{\text {off }}$ is observed at a peptide concentration of $0.6 \pm 0.4 \mathrm{mM}$, which is in agreement with the effect observed with $\mathrm{k}_{\text {on }}$ (Figure 2). This may indicate that both effects are caused by the same phenomenon, likely by the formation of the S1-peptide complex. From a chemistry point of view, this is possible if there are several binding sites for this peptide on the $\mathrm{S} 1$ protein, as one site must be occupied by the a1 domain of ACE2 in the process of complex formation.

Third, Figure 3 illustrates that preincubation of the peptide with the $\mathrm{S} 1$ protein before complex formation with ACE2 destabilized the S1-ACE2 complex since all $k_{\text {off }}$ values for series $B$ were higher than the equivalent values in series A. Importantly, this effect did not depend on the peptide presence, as observed when comparing the data points at zero peptide concentration. However, no systematic differences were observed in the binding experiments, performed with both preincubated and non-preincubated S1 protein samples at zero peptide concentration (Figure 2). Thus, the different stabilities of the S1-ACE2 complexes, with or without preincubation of the peptide with S1 protein, seem to be specific for the offrate reaction, however, the reasons for this phenomenon remain unclear.

Last, although the rate constants $\mathrm{k}_{\text {on }}, \mathrm{k}_{\mathrm{ass}}$, and $\mathrm{k}_{\text {off }}$ depend on peptide concentration (Figures 2 and 3 ), there is practically no influence of the peptide on the $K_{d}$ value, calculated as the ratio of the rate constants $\mathrm{k}_{\text {off }} / \mathrm{k}_{\text {ass. }}$. Thus, these data demonstrate that even effective peptide interaction with the S1 protein may not shift the equilibrium of the $S 1$ protein binding to ACE2. However, the peptide has a significant effect on S1-ACE2 complex formation and dissociation kinetics. This is an important 
conclusion to be considered in antiviral therapeutics design, as the simple inhibition mechanism of the virus-receptor binding process by peptides suggested in many papers seems to be oversimplified.

\section{Alternative peptide binding sites on the $\mathrm{S} 1$ protein}

The hypothesis that additional (allosteric) binding sites exist on the S1-ACE2 complex, which may bind additional peptide molecules that cause the "memory" effect in the off-rate experiments, was investigated computationally by mapping the putative docking landscape outside the known ACE2 binding site on the S1 protein. These calculations revealed that there are allosteric binding possibilities for the peptide QAKTFLDKFNHEAEDLFYQ (Figure 4).

It is important to emphasize that dissociation of the S1-ACE2 protein complex was initiated by transfer of the biosensor into buffer that did not contain peptide as well as S1. However, it can be seen in Figure 3 that the $\mathrm{k}_{\text {off }}$ value depends on the peptide concentration, which was used in the complex formation reaction, demonstrating "memory" relating to the peptide presence in the latter process. These results raise the following questions about the S1-ACE2 complex formation and its structure.

First, the occurrence of such "memory" indicates that different complexes could be formed in the presence of different peptide concentrations, or more likely, the peptide remains in some fraction of this complex, affecting its stability when compared with the binary complex.

Second, half of the peptide's effect on $\mathrm{k}_{\text {off }}$ is observed at a peptide concentration of $0.6 \pm 0.4 \mathrm{mM}$, which is in agreement with the effect observed with $\mathrm{k}_{\text {on }}$ (Figure 2). This may indicate that both effects are caused by the same phenomenon, likely by the formation of the S1-peptide complex. From a chemistry point of view, this is possible if there are several binding sites for this peptide on the S1 protein, as one site must be occupied by the a1 domain of ACE2 in the process of complex formation.

Third, Figure 3 illustrates that preincubation of the peptide with the $S 1$ protein before complex formation with ACE2 destabilized the S1-ACE2 complex since all $k_{\text {off }}$ values for series $B$ were higher than the equivalent values in series A. Importantly, this effect did not depend on the peptide presence, as observed when comparing the data points at zero peptide concentration. However, no systematic differences were observed in the binding experiments, performed with both preincubated and non-preincubated S1 protein samples at zero peptide concentration (Figure 2). Thus, the different stabilities of the S1-ACE2 complexes, with or without preincubation of the peptide with S1 protein, seem to be specific for the offrate reaction, however, the reasons for this phenomenon remain unclear.

Last, although the rate constants $\mathrm{k}_{\mathrm{on}}, \mathrm{k}_{\mathrm{ass}}$, and $\mathrm{k}_{\text {off }}$ depend on peptide concentration (Figures 2 and 3 ), there is practically no influence of the peptide on the $K_{d}$ value, calculated as the ratio of the rate constants $\mathrm{k}_{\text {off }} / \mathrm{k}_{\text {ass. }}$. Thus, these data demonstrate that even effective peptide interaction with the $\mathrm{S} 1$ protein may not shift the equilibrium of the $S 1$ protein binding to ACE2. However, the peptide has a significant effect on S1-ACE2 complex formation and dissociation kinetics. This is an important 
conclusion to be considered in antiviral therapeutics design, as the simple inhibition mechanism of the virus-receptor binding process by peptides suggested in many papers seems to be oversimplified.

\section{Alternative peptide binding sites on the $\mathrm{S} 1$ protein}

The hypothesis that additional (allosteric) binding sites exist on the S1-ACE2 complex, which may bind additional peptide molecules that cause the "memory" effect in the off-rate experiments, was investigated computationally by mapping the putative docking landscape outside the known ACE2 binding site on the S1 protein. These calculations revealed that there are allosteric binding possibilities for the peptide QAKTFLDKFNHEAEDLFYQ (Figure 4).

\section{Methods}

Peptide synthesis. The peptide QAKTFLDKFNHEAEDLFYQ was synthesized on an automated peptide synthesizer (Biotage Initiator + Alstra, Sweden) using the fluorenylmethyloxycarbonyl (Fmoc) solid-phase peptide synthesis strategy and Rink-amide ChemMatrix resin (PCAS- BioMatrix, Québec, Canada) to obtain C-terminally amidated product. $\mathrm{N}, \mathrm{N}^{\prime}$-diisopropylcarbodiimide (DIC) and Oxyma Pure in dimethylformamide (DMF) were used as coupling reagents, and $\mathrm{N}, \mathrm{N}$-diisopropylethylamine (DIEA) was used as the activator base. Cleavage of the product was performed with trifluoroacetic acid (TFA), $2.5 \%$ triisopropylsilane (TIPS), and $2.5 \%$ water for three hours at room temperature.

The peptide was purified by high-performance liquid chromatography (HPLC) on a C4 column (Phenomenex Jupiter C4, $5 \mu \mathrm{m}, 300 \AA, 250 \times 10 \mathrm{~mm}$, Agilent) using an acetonitrile/water gradient containing $0.1 \%$ TFA. The purity of the peptide was validated at $98 \%$ using a Waters Acquity UltraPerformance Liquid Chromatography (UPLC) with an acetonitrile/water gradient (Supplement Figure S1). The accurate molecular weight of the peptide was determined to be $2342 \mathrm{Da}$ using a matrix-assisted laser desorption/ionization time-of-flight (MALDI-TOF) mass spectrometer (Brucker Microflex LT/ SH, USA), with a-cyano-4-hydroxycinnamic acid as the matrix (Supplement Fig S2). The calculated molecular weight of the peptide was 2342.15 Da.

Proteins. Human recombinant ACE2-His protein (Icosagen OÜ, Estonia, cat\# P-302-100) and SARS-CoV-2 Spike protein S1 (Icosagen OÜ, Estonia, cat\# P-305-100) were used in this study.

Bio-Layer Interferometry (BLI). His-tagged ACE2 was immobilized onto Octet RED96e biosensors (ForteBio, CA, USA) and the binding of S1 protein was measured in the presence or absence of peptide QAKTFLDKFNHEAEDLFYQ. Experiments were performed at $25^{\circ} \mathrm{C}$ in $20 \mathrm{mM}$ Tris- $\mathrm{HCl} \mathrm{pH} 7.0$ and $150 \mathrm{mM}$ $\mathrm{NaCl}$. Biosensors (HIS1K, lot 6110102) were loaded with His-tagged ACE2, before S1 protein or S1 protein and peptide were added to start the complex formation process. In one series of experiments, the peptide was preincubated with $\mathrm{S} 1$ for $15 \mathrm{~min}$ at $25^{\circ} \mathrm{C}$ before the binding assay. Complex dissociation was initiated by immersing the biosensors into fresh assay buffer $(20 \mathrm{mM} \mathrm{Tris}-\mathrm{HCl} \mathrm{pH} 7.0$ with $150 \mathrm{mM} \mathrm{NaCl})$, without S1 protein and peptide. 
Data were analyzed using ForteBio Data Analysis software (version 11.1.1.39) [11]. Results are presented in the Supplement Table S1 and Table S2.

Computational peptide docking. The peptide docking study was performed as described previously $[5,6]$. Briefly, the input file for modeling the S1-ACE2 complex was built from X-ray analysis data [7, 9] deposited in the PDB database (www.wwPDB.org) with the code 6LZG. GROMACS package (version 4.6.1) was used for molecular dynamics simulations [14] and AutoDock Vina (version 1.1.2) was used for ligand docking [15]. The best scores were selected for peptide positioning. Protein protonation at $\mathrm{pH} 7 \mathrm{was}$ processed using the GROMACS pdb2gmx tool, and the geometric, charge, and van der Waals constrained parameters were assigned using the GROMOS 53a 6 force field parameter set [16]. The protein structure, neutralized by adding $\mathrm{Na}^{+}$and $\mathrm{Cl}^{-}$ions, was solvated in a $5 \mathrm{~nm}$ cubic box, filled with SPC water as solvent [17]. The system was allowed to reach equilibrium at constant pressure (1 atm) [18] and temperature $(300 \mathrm{~K})$, controlled by the modified Berendsen thermostat algorithm [19]. Equilibrated simulations were performed on the systems for ten nanoseconds. After MD relaxation, the protein structure was extracted from the system and used for docking procedures. The docking compatible structure formats of the protein were prepared by AutoDockTools (version 1.5.6) [20]. The fitting box with $0.3 \AA$ of grid spacing was defined once and used for all docking calculations. The fitting area covered the whole protein space and the docking poses were obtained and listed following the docking energy values. The graphic software package VMD (version 1.9.4) was used to illustrate ligand docking poses on the protein surface [21].

\section{Declarations}

\section{Acknowledgements}

Computational analysis was performed in the High-Performance Computing Center, and the peptide was synthesized in the Core Laboratory of Peptide Chemistry of the University of Tartu.

This work was financially supported by QanikDX OÜ, Estonia, registration number 4523084, grant LLTLT20014.

\section{Author contributions}

P.A., H.H and E.J performed experiments and analyses, A.K. performed computations, J.J., Ü.L. and M.U. planned this study and made data analysis. All authors wrote and reviewed this manuscript.

\section{Competing interests}

The authors declare no competing interests.

\section{References}

1. Shang, J., et al. Structural basis of receptor recognition by SARS-CoV-2. Nature 581, 221-224 (2020). 
2. Song, W., Gui, M., Wang, X., Xiang, Y. Cryo-EM structure of the SARS coronavirus spike glycoprotein in complex with its host cell receptor ACE2. PLOS Pathog. 14(8):e1007236 (2018).

3. Wu, C., et al. Analysis of therapeutic targets for SARS-CoV-2 and discovery of potential drugs by computational methods. Acta Pharm. Sin. B. 10, 766-788 (2020).

4. Han, Y., Král, P. Computational design of ACE2-based peptide inhibitors of SARS-CoV-2. ACS Nano 14, 5143-5147 (2020).

5. Kuznetsov, A., Järv, J. Mapping of ACE2 binding site on SARS-CoV-2 spike protein S1: docking study with peptides. Proc. Est. Acad. Sci. 69, 228-234 (2020).

6. Kuznetsov, A., Jarv, J. Mapping of ACE2 binding site on SARS-CoV-2 spike protein S1: Molecular recognition pattern. Proc. Est. Acad. Sci. 69, 355-360 (2020).

7. Wang, Q., et al. Structural and functional basis of SARS-CoV-2 Entry by Using Human ACE2. Cell 181, 894-904 (2020).

8. Yan, R., et al. Structural basis for the recognition of SARS-CoV-2 by full-length human ACE2. Science 367, 1444-1448 (2020).

9. Lan, J., et al. Structure of the SARS-CoV-2 spike receptor-binding domain bound to the ACE2 receptor. Nature 581, 215-220 (2020).

10. Frenzel, D., Willbold, D. Kinetic Titration Series with Biolayer Interferometry. PLOS ONE 9, e106882 (2014).

11. Tobias, R., Kumaraswamy, S. Biomolecular Binding Kinetics Assays on The Octet Platform. Application note14. ForteBio, Pall Life Sciences, http://www.biophysics.bioc.cam.ac.uk/wpcontent/uploads (2011).

12. Reaction Biology. SARS-CoV-2 S Protein and ACE2 Binding Assay.https://www.reactionbiology.com/services/covid-19-related-assays (2020).

13. Benton, D., J. et al. Receptor binding and priming of the spike protein of SARS-CoV-2 for membrane fusion. Nature 588, 327-330 (2020).

14. Hess, B., Kutzner, C., van der Spoel, D., Lindahl, E. GROMACS 4: algorithms for highly efficient, loadbalanced, and scalable molecular simulation. J. Chem. Theory. Comput. 4, 435-447 (2008).

15. Trott, O., Olson, A., J. AutoDock Vina: improving the speed and accuracy of docking with a new scoring function, efficient optimization, and multithreading. J. Comput. Chem. 31, 455-461 (2010).

16. Oostenbrink, C., Villa, A., Mark, A., E., van Gunsteren, W., F. A biomolecular force field based on the free enthalpy of hydration and solvation: The GROMOS force-field parameter sets 53A5 and 53A6: J. Comput. Chem. 25, 1656-1676 (2004).

17. Berendsen, H., J., C., Grigera, J., R., Straatsma, T., P. The missing term in effective pair potentials. J. Phys. Chem. 91, 6269-6271 (1987).

18. Parrinello, M., Rahman, A. Polymorphic transitions in single crystals: A new molecular dynamics method. J. Appl. Phys. 52, $7182-7190$ (1981). 
19. Berendsen, H., J., C., Postma, J., P., M., van Gunsteren, W., F., DiNola, A., Haak, J., R. Molecular dynamics with coupling to an external bath. J. Chem. Phys. 81, 3684-3690 (1984).

20. Morris, G. et al. Autodock4 and AutoDockTools4: automated docking with selective receptor flexibility. J. Comput. Chem. 16, 2785-2791 (2009).

21. Humphrey, W., Dalke, A., Schulten, K. VMD - visual molecular dynamics. J. Mol. Graph. 14, 33-38 (1996).

\section{Figures}

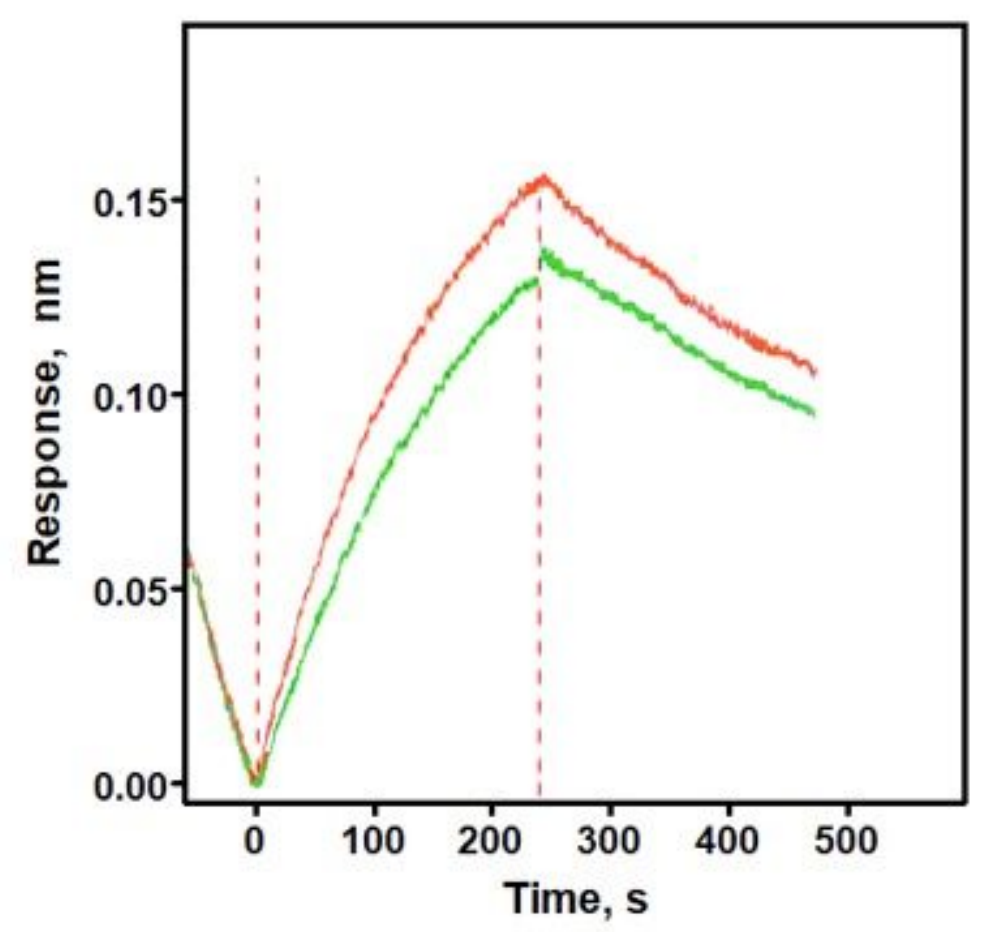

\section{Figure 1}

Kinetic curves, characterizing the time course of SARS-CoV-2 spike protein S1 binding to ACE2 protein loaded onto the biosensors of the instrument (ascending curve) and dissociation of this complex (descending curve). Red line: experiment performed using the assay buffer without the peptide. Green line: experiment performed in the presence of $5 \mathrm{mM}$ peptide QAKTFLDKFNHEAEDLFYQ, which had been preincubated with the SARS-CoV-2 spike protein S1 for 15 minutes before the assay. 


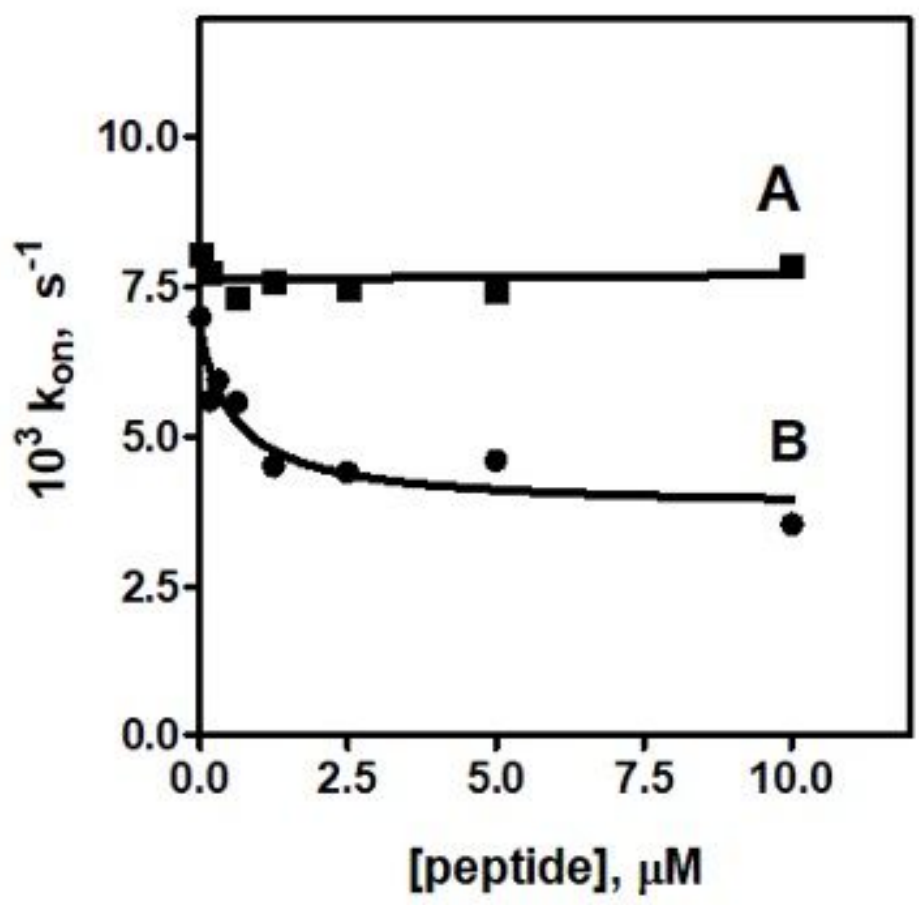

Figure 2

The influence of the peptide QAKTFLDKFNHEAEDLFYQ on the rate constant (kon) of S1 binding to ACE2, where ACE2 is immobilized on the biosensor. A. Spike protein S1 and peptide were simultaneously added to the assay buffer before the binding assay was initiated (squares). B. Spike protein S1 was preincubated with the peptide for $15 \mathrm{~min}$ in the assay buffer before the binding assay was initiated (circles). 


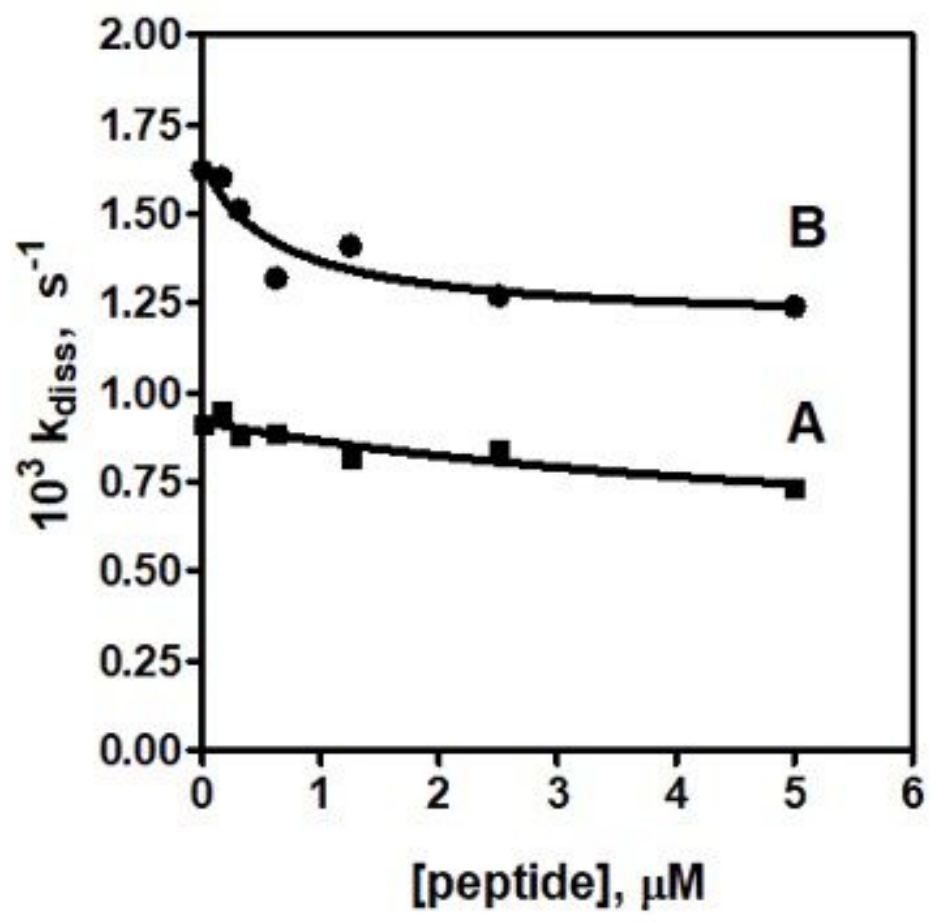

\section{Figure 3}

Dissociation of the S1-ACE2 complex, captured by the biosensor in the binding assay. The S1-ACE2 protein complex was formed in the presence of different peptide concentrations in experiments where the $\mathrm{S} 1$ protein had been preincubated with the peptide for 15 minutes (series $\mathrm{A}$, squares), or the $\mathrm{S} 1$ protein had not been preincubated with the peptide (series B, circles). To initiate the dissociation process, the biosensor was transferred into fresh buffer that did not contain peptide and S1 protein.

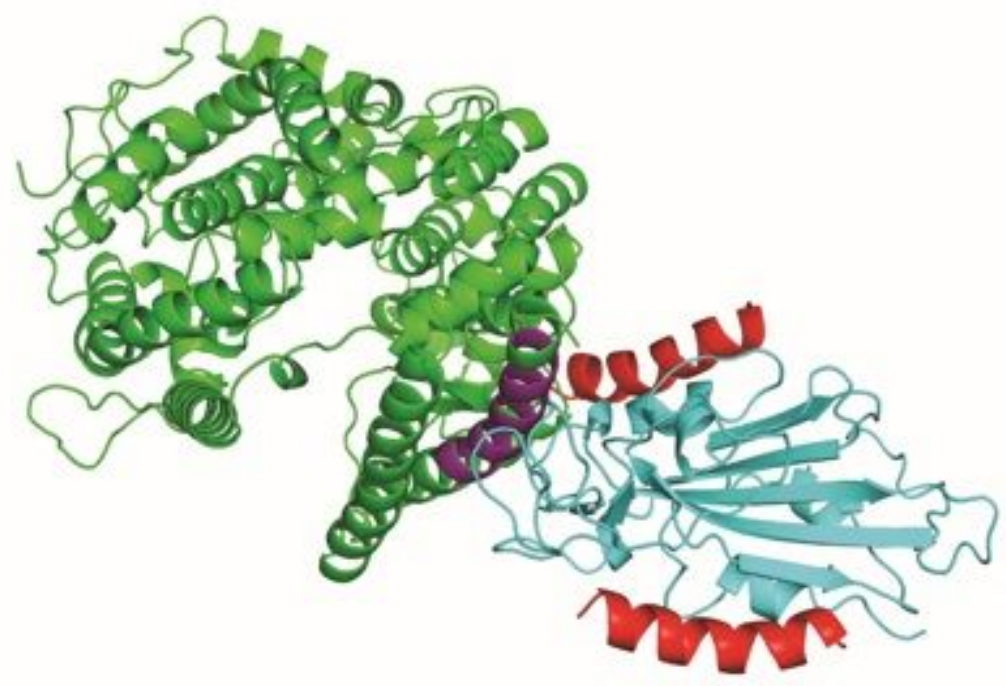


Figure 4

Ribbon structure of the complex formed between the SARS-Cov-2 spike protein S1 and ACE2. The S1 protein is shown in blue and the ACE2 molecule is shown in green. Two allosterically bound peptide QAKTFLDKFNHEAEDLFYQ molecules are shown in red. The sequence (amino acids 24-42) of the a1 domain of ACE2 which interacts with the $\mathrm{S} 1$ protein is shown in violet.

\section{Supplementary Files}

This is a list of supplementary files associated with this preprint. Click to download.

- SupportingFiguresandTables.pdf 